\title{
When Loneliness Evolves into Solitude: The Answer to the Self from Within
}

\author{
Kuo Bian \\ University of Illinois at Chicago, Chicago, USA \\ Email: biankuo9511@gmail.com
}

How to cite this paper: Bian, K. (2021). When Loneliness Evolves into Solitude: The Answer to the Self from Within. Open Journal of Philosophy, 11, 620-631. https://doi.org/10.4236/ojpp.2021.114038

Received: October 28, 2021

Accepted: November 27, 2021

Published: November 30, 2021

Copyright (C 2021 by author(s) and Scientific Research Publishing Inc. This work is licensed under the Creative Commons Attribution International License (CC BY 4.0).

http://creativecommons.org/licenses/by/4.0/

\begin{abstract}
Loneliness and solitude have similar attributes to individuals, but there is a critical variance between the two regarding the impact on the individual. Loneliness is an unpleasant feeling in a broad sense, while according to some philosophers, solitude is regarded as a joyful necessity when one establishes a deep relationship with the outside world. This article aims to develop a sensible account of the difference between solitude and loneliness by looking at some insightful philosophical viewpoints. Importantly, this article aims to explain the dimensions in which solitude is a positive phenomenon. I will analyze the role of solitude in eliminating loneliness from two aspects: self-consciousness and self-knowledge construction.
\end{abstract}

\section{Keywords}

Loneliness, Solitude, Style, Self Conscious, Styling, Insert

\section{Introduction}

The search for a solution to the modern loneliness problem has gradually become blurred, and some recent arguments will confuse it further. This is because the interaction between individuals in a diverse context has become complicated and radical.

Amelia Worsley noted in "A History of Loneliness" that "in 1674, the naturalist John Ray compiled a glossary of infrequently used words." He included "loneliness" in his list, defining it as a term used to describe places and people "Far from neighbors" (Worsley, 2020). An "uncommon vocabulary" and a rare social isolation phenomenon are the first impressions of loneliness in the past. Back to modern times, in 2017, the Joe Cox Loneliness Committee submitted a report to the British Parliament stating that "more than 9 million people in the country often or always feel lonely (Ceylan, 2018).” In January of the following year, the 
United Kingdom appointed Tracy Crouch, the Deputy Minister of Sports and Civic Affairs, as the "Lonely Minister" to lead a government team focused on solving the problem of social loneliness. When this amazing title of "Lonely Minister" was established, it marked that loneliness has officially become a humanistic psychological problem that needs to be solved by government leaders in this impetuous and interactive era.

In the general sense, people will not feel excessively repelled by the official position of "Lonely Minister" because, for lonely people, an unpleasant and unrecognized emotion haunts them. However, imagine if the United Kingdom appoints a secretary for the problem of "Solitude", people will likely raise doubts about the meaning of this title; does solitude mean loneliness? Is being alone a problem? Obviously, people have not reached a clear understanding of loneliness and solitude in their cognition. The reason is that they might not be sure if it is natural to explain solitude in terms of loneliness by looking at John Ray's definition, or by their first sense of loneliness.

That incident raises an intriguing question, what is the difference between loneliness and solitude? When discussing loneliness and solitude, we need to start from the inside of ourselves and evaluate the changes in one's relationship with the outside. Therefore, it is appropriate for solitude and philosophy to have a fierce collision. I aim to make a philosophical comparison between solitude and loneliness and show the correlation between both. Importantly, this article intends to explain the positivity of solitude for individuals by clarifying the features of solitude.

\section{A Philosophic View of Loneliness}

\subsection{Reorganizing Dimensions of Loneliness}

Philosophy's interpretation of loneliness is not difficult to understand. Philip Koch, in the book "Solitude: A Philosophical Encounter", understands loneliness as a kind of sentiment analysis:

Loneliness is the unpleasant feeling of longing for some kind of interaction with another person(s). The desire for others may be driving or mild, specifically focused or vague, self-conscious or unnoticed, which others and what is needed from them can remain unclear in the emotion. But the yearning for others is the core of this painful emotion: what it seeks is a healing personal engagement (Koch, 1997: 185).

We first need to be aware that loneliness is a negative emotion in which we are unconnected with the outside world. "Emotions are things you feel, though they are not simply bodily feelings. Additionally, they are structures of belief and evaluation (Koch, 1997: 31)." To a large extent, in Koch's account, loneliness is dissatisfaction between the self and the external world. When someone feels lonely, an unbalanced negative emotion that arises when self-belief evaluates the relationship with the outside. Thus, the two necessary conditions for loneliness are a self with consciousness and an external world with other consciousness, 
which includes the different consciousness of others.

Nevertheless, we need to maintain a cautious attitude. The emotion here referring a sensory consciousness that involves an individual's negotiation with the outside world. Thus, I think it is difficult for us to distinguish this emotion's nature. The reason is that if loneliness is attributed to a kind of emotion, it is difficult to interpret whether I caused my loneliness or someone else caused my loneliness. In that event, we won't distinguish whether subjective or objective reasons cause loneliness. When we deal with the problem of loneliness, human beings are expected to make corresponding consciousness and physical actions to reconcile or choose between the self-state and the external world. These two concepts of active and passive reflect two different phenomena; I think that people isolate me and others isolate me. Thus, we need to start from the individual's interior to think about the changes in one's relationship with the outside to find the causation.

Empiricists maintain that the human world is gradually constructed based on the interaction between the self-subject and the external world. As John Locke noted in "Thoughts Concerning Education" that "We are all a sort of chameleons, that still take a tincture from things near us: nor is it to be wondered at in children, who better understand what they see, than what they hear (Locke, 1963: 32)." Our vision is formed based on a self-formative approach, which is highly subjective. It is true that the limitations of people and society indeed determine that people must cooperate with other groups in society to survive. When the self and the outer world are in a kind of conflictive state, loneliness appears as a pronoun. However, when the self interacts with the outside world, the ambiguity of the subject-object relationship makes it difficult to determine which consciousness phenomenon is the suitable way for the self to manage loneliness. Trying to solve the adverse psychological problems caused by loneliness from the emotional level is an imprecise way, and it is far from enough for our "Lonely Minister" to solve the problem of the soaring suicide rate of modern people. Accordingly, loneliness needs to be interpreted in particular situations, and therefore, we should dig out every possible causation that led to loneliness. Here I propose four underlying circumstances that lead to loneliness:

1) Incompatibility between self-knowledge and the outside world; in the social category, people with different visions will have different resonances in communication. When the external environment and self-awareness become incompatible, loneliness will appear. For example, if a Chinese who does not speak English lives in an English environment, the consciousness of the Chinese will probably not be effectively shared, given that this Chinese is not sufficient at expressing his views. Thus, it will likely lead to a state of loneliness. Imagine a person who has passively lost the ability to interact with the outside world. It is hard not to feel alone. By all means, I am referring to English skills as knowledge-level incompatibility, which may also occur in other levels of knowledge incompatibility, such as social culture, religious culture, and differences in opinions. 
2) Personality hedges; personal character is determined based on different economic, cultural, and even biological backgrounds (etc.). Different personalities are also destined to be unable to have permanent peace and harmony between individuals. In the modern context, although society's approach to diversity has endorsed an attitude of equality and respect, it still cannot solve the barriers caused by differences in personal characteristics. This kind of natural isolation will cause isolation between individuals, thereby creating a sense of loneliness in a shared environment. For example, "Out-of-group" will be rejected in the public domain. If homosexuality is not recognized by society and the government, homosexuality is the lonely party in the social group. This kind of "discrimination" is caused by personality differences, which creates loneliness in different groups.

3) The imbalance between the self and the external world; Schopenhauer stated with respect to the imbalance between the self and the external world in "Studies in Pessimism" that "Every man takes the limits of his own field of vision for the limits of the world (Schopenhauer \& Saunders, 2014: 20)." What I think is different from what you think" is a sense of opposition that undoubtedly brought confrontations and divisions within the social sphere. Simply put, there is no continuous commonality between individuals. Excluding the idea of social etiquette and multicultural identity, an internal contradiction has become the origin of loneliness. It is crucial to notice that individuals first evaluate a feeling of oneself rather than correctness when they construct self-awareness. A sense of inferiority or arrogance is motivated when their consciousness is not proportional to the feedback from the outside world. This kind of self-appreciation or self-denial brings the funny question of whether I do not understand the world, or the world does not understand me? A sense of self-unawareness potentially introduces individual consciousness into the conflict with the external world, which creates a sense of loneliness among confused people. We are not here to intend to criticize people who are ignorant or arrogant because the cause of arrogance is still due to the inconsistency between the self and the external world. Before people realize this, the consciousness of incomprehension will be led by a sense of loneliness.

4) Tragedy and regret, in the movie "The Great Gatsby", Scott once said that "The loneliest moment in someone's life is when they are watching their whole world fall apart, and all they can do is stare blankly" (Scott Fitzgerald). Tragedy and loneliness are usually related. Tragedy is considered by us to be a disaster away from the principle of happiness. We regard a child whose parents have passed away as lonely because the particularity of the disaster makes the child lose the ability to manage loneliness from the root. A father who is not cared for by his children, we think he is lonely because the emotional power he wishes to have has become a regret in his life.

\subsection{Nowhere to Escape Loneliness}

Despite which of the above factors, or the loneliness based on which kind of in- 
formation asymmetry, it mirrors Koch's distinction of loneliness: loneliness is a negative emotion that cannot be longed for. However, the emotion of loneliness is based on multiple situations in a way involving the disconnection of personal consciousness from the outside world. The natural imbalance, incompatibility, and hedging between the individual will and the external world potentially create a feeling of unpleasantness. This kind of negative emotion finally caused loneliness in the context of an era that constantly requires interactivity. By carefully exploring the four factors of loneliness, we could find that the conflicts behind loneliness are irreversible. In the context of modern pluralistic democratization, individual consciousness has become an open debate. We are told and allowed to have various thoughts, but we are not learning well to deal with the conflicts between our consciousness and the external world. The incompatibility and imbalance between the self and the external world all play their respective roles under the concept of pluralism. It is not hard to imagine that lonely people often think it is not easy to find an ideal communication partner. Suppose we understand this state from the individual consciousness. We may conclude that the destined split between people also indicates the destined sense of loneliness, which will permeate the individual and the external world forever. Thus, we shall make a simplified summary here: "Conflicts" is the essence behind loneliness. A conflict refers to a state of conflict between oneself and the world, which results into a state of loneliness. On the one hand, the self is the only subject who feels lonely because loneliness is an emotion for an individual, indicating an individual's internal factor. On the other hand, we build our self-awareness based on not always being in perfect harmony with the outside world. Conflict is the contradiction between the self and the external world, which involves a kind of mismatch between an individual's knowledge, experience, and desire and, so on. A human constructs his own perceptions, emotions, bodily sensations, memories, and beliefs in the way of self-informative. In this process, individuals will realize that they are not a unitary existence at the same time based on the influence of context. One's enemies, friends, and colleagues do not have the expected conditions to share the same "self" with me because they do not have the exact attributes with me. Consequently, such a non-equal information exchange caused conflicts.

\subsection{The Dilemma of Loneliness in Modernity}

The conflict is further explained and clarified more radically from the perspective of philosophers. In "Studies in Pessimism: A Series of Essays", Schopenhauer described the connection between the individual and the external world in this way:

In early youth, as we contemplate our coming life, we are like children in a theatre before the curtain is raised, sitting there in high spirits and eagerly waiting for the play to begin. It is a blessing that we do not know what is really going to happen. Could we foresee it, there are times when children might seem like 
innocent prisoners, condemned, not to death, but to life, and as yet all unconscious of what their sentence means. Nevertheless, every man desires to reach old age, in other words, a state of life of which it may be said: "It is bad today, and it will be worse tomorrow, and so on till the worst of all (Schopenhauer \& Saunders, 2014: 3)."

As Schopenhauer has attached the tragic color to the conflict between oneself and the world; a helplessness feeling is caused along with the fall of the individual's vision. When a contradiction cannot be resolved, breaking or reorganizing a confliction sometimes would be an irrational solution. War emerges as an irrational solution when there is an unresolvable conflict between states. When unbreakable conflicts occur between people, a rupture becomes a common means to avoid further conflict. Similarly, when loneliness cannot be avoided through personal normative behavior, reorganizing the definition of loneliness has become the interest of philosophers. However, in my opinion, this interest does not spell out the definitions of loneliness and solitude clearly in terms of the loneliness problem of modernity. Such pessimistic philosophical thinking did not try to treat the pain of loneliness rationally but tragicized loneliness into an irresolvable dilemma. Rudolph Kipling once said that "The individual has always had to struggle to keep from being overwhelmed by the tribe. If you try it, you will be lonely often and sometimes frightened. But no price is too high to pay for the privilege of owning yourself (Gordon, 1967).” The label of pessimistic philosophy is inseparable from loneliness. While philosophers claim to accept the inevitable loneliness, they bring the individual into an inevitable lonely circle. And with that kind of viewpoint, they are portraying loneliness as an aesthetic tragedy;

What a person is for himself, what abides with him in his loneliness and isolation, and what no one can give or take away from him, this is obviously more essential for him than everything that he possesses or what he may be in the eyes of others (Schopenhauer, 2014: 5).

Oneself have tried to dissolve loneliness, but one finds that the sense of loneliness is inevitable, so we shall claim that loneliness will always be with me. Understandably, Pessimists believe that loneliness is insoluble. Their declaration of loneliness represents the sentiment of accepting conflict while enjoying their "loneliness". I think this is an imprecise and irresponsible judgment criterion that blurs the definition of loneliness and solitude. Do they enjoy loneliness or solitude? How should the difference be defined?

Hence, the concepts of accepting loneliness and enjoying loneliness need to be further distinguished. Loneliness should be reconciled through a personal will. Based on the conclusions we have drawn on loneliness; time cannot be reversed, regrets cannot be rejected, and the differences between individuals cannot be reset. When the complexity of the external world blocks the exit of loneliness, loneliness will eventually lead to examining our self-will.

\section{The Different Dimensions of Loneliness and Solitude}

In "Why I Am So Wise", Nietzsche noted that "My humanity is a constant 
self-overcoming, but I need solitude: a recovery, a return to myself, a breath of a free, light, playful air. The whole of my Zarathustra is a dithyramb to solitude, or if you have understood me, to purity (Nietzsche, 2009: Chapter 3).” The idea of self-will is an effective way to solve the problem of loneliness; individuals need to improve the quality and resilience of self-awareness in a conflicting environment to be more tolerant of conflicts. In this logic, the essence of solitude is revealed in the way of self-reflection. Therefore, distinguishing and analyzing the difference between loneliness and solitude has become the key to exploring solutions. Koch gave a comprehensive explanation of solitude. The three characteristics of solitude he proposed are Physical Separation, Social Disengagement, and Reflectiveness (Koch, 1997: 184). Here, physical separation aims at separation in the physical sense, and social alienation aims at separating consciousness from the outside world, which all point to the characteristics of loneliness we mentioned above. It is worth noting that the two specific terms of Koch point out the fundamental difference between solitude and loneliness; solitude is an autonomous choice, which is primarily based on subjectivity. Loneliness is a conscious phenomenon that combines objective and subjective phenomena. When the individual is in a state of solitude, the individual consciousness is actively isolated from the external world. As Audrey Hepburn said, "I have to be alone very often. I'd be quite happy if I spent from Saturday night until Monday morning alone in my apartment. That's how I refuel (Audrey, 1953)." Hepburn's choice expresses strong self-will here. The term "alone" is embodied in the subjective separation of the body and consciousness from the outside at the same time. Loneliness does not have such apparent personal distinction, according to our analysis above.

\subsection{Reflectiveness as Core of Solitude}

Positioning as of reflectiveness, it is the special right and property of the individual in the solitude process in Koch's account. "For solitude is not captured well in the simple phrase "exist alone"; after all, loneliness, isolation, and alienation are also ways of existing alone, yet none of these seem quite the same as solitude (Koch, 1997: 182)." The separation of body and consciousness from the outside world can't be pointless. If Hepburn only stops her consciousness from Saturday to Monday, it will become a meaningless individual stagnation. This valueless separation phenomenon would like sleeping and death, which does not fit with the characteristics of solitude. Therefore, reflectiveness in solitude here points to a reflection within consciousness.

1) A person in solitude will actively reject the interaction with the outside world. This is the order of physical isolation and then consciousness isolation.

2) Meanwhile, I retained all my will from birth to the present and let it interact internally with my exhaustive knowledge of the world.

3) The establishment of internal reflection allows the meaning of solitude to be established. Otherwise, solitude will be relegated to a meaningless escape and 
protest by those who are feeling lonely.

"A solitude is a stretch of experience disengaged from other people in perception, thought, emotion, and action (Koch, 1997: 57)." Therefore, solitude is a state of being alone in which consciousness and body are actively isolated from the external world while simultaneously combing reflecting on all their willpower.

Moreover, Koch noted that "but not yet clear or subtle enough; for there are modes of diminished engagement, thus far unexplored, which have the greatest importance for understanding the experience of solitude (Koch, 1997: 57)." Here is a particular situation that needs to be considered; since solitary reflection belongs to internal review and consciousness is a feature that cannot be perceived and passively shared, whether the individual can only isolate consciousness while not isolating the body? This is indeed a unique situation. In my understanding, those who can handle consciousness and external negotiations separately can be called "solitary masters" or "emotional solitude." But in a broad sense, communication is a prerequisite for consciousness to interact, and most people cannot isolate consciousness separately without communicating with the outside. If Hepburn can separate consciousness when she negotiates with the outside world, she won't need to spend Saturday to Monday to refuel herself. Hepburn may not be a master of solitude, needs to seek the peace of consciousness in this impetuous and prosperous world, as most people would do. The flowers and praise of the outside world indeed belong to Hepburn; however, seeking to release the exhaustion caused by dealing with the complex world is the original intention of she's solitude.

Thoreau in "Walden" noted that "I would rather sit on a pumpkin and have it all to myself than be crowded on a velvet cushion (Thoreau, 1995: Chapter Economy, 55)." When the individual and the person will are in a parallel and uninterrupted state, we can truly think about what belongs to us, including our own analysis of happiness, pain, value, will, and so on. Modern people are often confused by the questions are: Who am I? What do I have? What do I want? Being alone may not be able to answer these questions promptly, but it is indeed a quiet beginning without external interference. Different from loneliness, solitude suspends the unpleasant emotions caused by self-external conflicts and seeks self-salvation.

\subsection{Reflectiveness as a Transition between Solitude and Loneliness}

The meaning of reflexivity for solitude is beyond internal reflection, which is more inclined to the sorting and sublimation of consciousness. John Perry's theory of self-knowledge would be an ideal fit with the reflectiveness of solitude. In Perry's theory of self-knowledge, a buffer zone of the self is used as a medium for collecting and organizing external information. As Neil Leeuwen's analysis in "Perry on Self-Knowledge" that "a perceptual buffer is just a firststory notion/file that tracks information about an object (it could be oneself) 
through one or more perceptual channels (Leeuwen, 2012: 13)." Under certain circumstances, the attachments of the self-buffer will be used to construct self-knowledge. For instance, when I have a dispute with someone, the information about the dispute will be recorded in a buffer of my notion and stored briefly. When I came into contact with the information related to this dispute again, the information of that dispute will be automatically extracted to this moment. By then the individual will integrate the new information with the old information and begin to think that these two types of pieces of information are based on one's self-will. At this point, self-reflection in a true sense has transcended the classification of subjective and objective factors, thus has achieved a state of realizing a judgment of the correctness of self-will. We do not need to worry about this reflective "correctness" because we can learn from the characteristics of solitude and reflection that the two are not a one-off event but phased self-organization. I may think that I was right in that dispute this year, and a few years later I will deny ourselves based on the new knowledge I have gained. However, whether I deny or insist on my position, my will always be with me, which is the only thing that can convince me that I am not alone. In "The Self, Self-Knowledge, and Self-Notions", Perry defines the meaning of self-buffer to self in this way:

There is only one person I will ever be identical with, myself. I never have to unlink my self-buffer from my John Perry notion. It can be a self-notion; it can just be my self-buffer. Accumulating information in one's self-buffer for life is valid, unlike accumulating in one's here buffer longer than one stays in one place (Perry, 2002: 28).

The idea of self-buffer gives reflection a progressiveness. It allowed one to separate their self-will from the external contradictions and turned it into a verification between the self and the outside. At this time, the negative emotions caused by conflict have found a peaceful habitat, in which solitude brings a relative balance between loneliness and self. Why do we define the reflective nature of solitude and the conflicting nature of loneliness to be connected here? This is because, in the logically closed loop of irreversible loneliness, we found a secluded path through solitude and got out of the shackles of conflict.

1) Reflectiveness occurs in the interaction between individuals and things.

2) Conflictions, imbalance, and incompatibility between the self and the outside world are caused by the interaction between the self and the outside.

3) The interaction with the outside satisfies the establishment of conflict, and the painful sense of loneliness arouses the individual's reflection on the self and the outside world.

4) This kind of reflection needs to be based on internal reflection in a state of isolation because if the individual does not stop interacting with information, there will only be endless conflicts and loneliness that follow.

Therefore, stopping the conflict and gradually reflecting on the current state is a shortcut to get out of the conflict wall. The first condition before a kind of doctrine can be criticized is that this doctrine needs to be created first and that a 
question needs to be reflected cannot be separated from the doubts and anxiety that the question brings to the individual. The reason why solitude can be distinguished from loneliness by reflection is that the lonely individual stimulates the operation of self-buffer in the process of constructing self-knowledge, so as to internally think about the conflicts caused by loneliness.

\subsection{Loneliness Evolving into Solitude}

Along with rigorous thinking, a logical flaw here still confuses the characteristics of solitude and loneliness; conflict is irreversible and continuous, which the self in the reflection in solitude will eventually be irreconcilable with the outside. Schopenhauer's pessimistic philosophy believes that loneliness is one of the forms of life. As he described in his works "Essays and Aphorisms" that "A man can be himself only so long as he is alone; and if he does not love solitude, he will not love freedom; for it is only when he is alone that he is really free (Schopenhauer, 2014: 20)." Conflict cannot be avoided as long as the individual is still negotiating with the outside, so the way to avoid loneliness is to separate the self from the outside properly. Only in this artistic conception, self-awareness and cognition can evolve into a free form that is not affected by the outside world. The reason for Schopenhauer's pessimistic attitude towards loneliness is precise because that the irreversibility of conflict leads to loneliness, which is inevitable and continuous for the individual. Even if the way of being alone can get peace in this, it is short-lived under the influence of this force majeure conflict environment. In any sense, we cannot let loneliness return to the vaguely defined model of loneliness based on modernity because, in this way, we return to the tragic aesthetics of loneliness.

The loneliness of modernity does not allow us to arbitrarily create the tragic color of its heroism. It is very simple that we need to express the meaning of solitude through philosophy so that the future and existing "Lonely Minister" can continue to work. Ned Vizzini noted in his book "It's Kind of a Funny Story" that "I didn't want to wake up. I was having a much better time asleep. And that's really sad. It was almost like a reverse nightmare like when you wake up from a nightmare, you're so relieved. I woke up into a nightmare (Vizzini \& Cohn, 2015: 15)." The police recognized this outstanding modern writer as having died of an apparent suicide. An aversion to survival caused by conflict cannot be cured by the tragic philosophy of loneliness. The problem of modern loneliness should not be regarded as a tragedy full of beauty and heroism. Suppose suicide becomes the ultimate solution to the problem of modern loneliness. In that case, the philosophy of loneliness is no longer an aesthetic philosophy of mind but a disease of the times. We might attribute lonely aesthetics to the work of literary scholars, who express the charm of aesthetics and literature to reorganize the core of tragedy. The world created by the times does not give people breathing space when they gather together, thus, the analysis of loneliness and solitude needs to be the responsibility of philosophy. 


\section{Conclusion}

Therefore, the individual can deal with the conflict brought about by the conflict in the way of solitude. I shall propose to let loneliness be converted to solitude; let people wandering in the loneliness be solitude in a peaceful manner. The reason is that solitude is different from loneliness, in which loneliness struggles with conflict, but solitude can reflect and sublimate in this circle struggle.

In the process of reflecting based on self-knowledge and external hedging, solitude turned the center of contradiction from the outside to the inside, thus realizing the internal digestion of self-consciousness. Under this premise, the individual in solitude degrades the conflict into a form of trying to contain, deliberating, reflection and trying to understand. This may be a short-lived utopian mood, but solitude builds individual consciousness on the basis of self-evolution and sublimation rather than being imprisoned in negative emotions caused by loneliness. As Albert Camus once analyzed being alone in "The Myth of Sisyphus and Other Essays" that:

In order to understand the world, one has to turn away from it on occasion; to serve men better, one has to hold them at a distance for a time. But where can one find the solitude necessary to vigor, the deep breath in which the mind collects itself and courage gauges its strength? There remain big cities. Simply, certain conditions are required (Camus, 1955: 96).

There remains an extensive undeniable external framework that is constantly causing conflicts and contradictions. Our loneliness needs some skills to be alleviated. Therefore, a more subtle self-isolation leads the individual towards positive reflection and learning between the self and the outside world. At this moment, loneliness evolved into solitude, liberating conflicts and creating a free and peaceful me when dealing with the outside world.

\section{Acknowledgements}

I would like to express my sincere gratitude to my mentor, John Perry, a Philosophy Emeritus Professor at Stanford University, who gave me the rare opportunity to explore this thoughtful project on the topic of solitude. My completion of this project could not have been accomplished well without his encouragement and support. When I sought a solid foundation on how to combine solitude and reflection, Perry's explanation of self-buffer enlightened me a lot. Besides, as an extraordinary writer, Professor Perry has given me a range of guidance during my writing process. He often accurately captured my imprudent thoughts, which enabled me to think about my topic more comprehensively. His humor and insights promoted my progress. It was a great comfort and enjoyment to communicate with him-my heartfelt appreciation.

\section{Conflicts of Interest}

The author declares no conflicts of interest regarding the publication of this paper. 


\section{References}

Audrey, H. (1953). A Quote by Audrey Hepburn. Goodreads. https://www.goodreads.com/quotes/160720-i-have-to-be-alone-very-often-i-d-be-quite

Camus, A. (1955). The Myth of Sisyphus: And Other Essays. Hamish Hamilton.

Ceylan, Y. (2018). U.K. Appoints a Minister for Loneliness. The New York Times. https://www.nytimes.com/2018/01/17/world/europe/uk-britain-loneliness.html

Gordon, A. (1967). Kipling on the Individual and the Tribe. The Kipling Journal. https://www.stephenhicks.org/2014/03/20/kipling-on-the-individual-and-the-tribe/

Koch, P. (1997). Solitude: A Philosophical Encounter. Open Court.

Leeuwen, N. V. (2012). Perry on Self-Knowledge. In A. Newen \& R. V. Riel (Eds.), Identity, language, and mind: Introduction to the philosophy of John Perry (pp. 89-107). CSLI Publications.

Locke, J. (1963). Some Thoughts Concerning Education. https://download.tuxfamily.org/openmathdep/teaching/Education-Locke.pdf

Nietzsche, F. W. (2009). Why I Am so Wise. Chartwell Books.

Perry, J. (2002). The Self, Self-Knowledge and Self-Notions. In Identity, Personal Identity, and the Self. Hackett Publishing. http://john.jperry.net/cv/2002cS\%2CSlfKN\%2CSlfNO.pdf

Schopenhauer, A. (2014). Essays and Aphorisms. Penguin Books Ltd.

Schopenhauer, A., \& Saunders, T. B. (2014). Studies in Pessimism: A Series of Essays. BiblioLife.

Thoreau, H. D. (1995). The Project Gutenberg eBook of Walden. https://www.gutenberg.org/files/205/205-h/205-h.htm\#chap02

Vizzini, N., \& Cohn, R. (2015). It's Kind of a Funny Story. Hyperion.

Worsley, A. (2020, July 31). A History of Loneliness. The Conversation. https://theconversation.com/a-history-of-loneliness-91542 